\title{
Twin-bunch compression via velocity bunching in a traveling wave accelerator
}

\author{
Dan Wang, ${ }^{*}$ Hongze Zhang, QiLi Tian, Dong Wang, YingChao Du, Lixin Yan, ${ }^{\dagger}$ Wenhui Huang, \\ Wei Gai, and Chuanxiang Tang \\ Department of Engineering Physics, Tsinghua University, Beijing 100084, China
}

(Received 11 June 2018; published 27 August 2018)

\begin{abstract}
The twin-bunch technique has been a subject of intense investigation due to its application in diverse fields, such as two-color free-electron lasers and beam-driven wakefield acceleration. In this paper, we investigate the generation of twin bunches (tunable in both time and energy space) via a velocity bunching (VB) scheme in a traveling wave accelerator (TWA) by varying the TWA phase across a very large range for the first time. The results of both simulations and experiments are presented. The results show that when the phase injected into the TWA tube is set to $\ll-100$ degrees, VB occurs in a deep overcompression mode, where the twin bunches are continuously and widely tunable under a large TWA phase acceptance. The energy and time spaces can be adjusted in the ranges [0-3] MeV and [0-6] ps, respectively, by varying the phase of the injected bunch train from -170 degrees to -120 degrees. The deep overcompression mode is a newly explored working mode for the VB scheme. Moreover, the emittance of each sub-bunch can be preserved when the strength of the long solenoid surrounding the TWA is optimized for emittance compensation, thus maintaining the high-quality beams from photoinjectors.
\end{abstract}

DOI: $10.1103 /$ PhysRevAccelBeams.21.084403

\section{INTRODUCTION}

The recent development of two-color x-ray free-electron lasers (FELs) [1-6], as well as the successful demonstration of high-gradient witness-bunch acceleration in wakefields (plasma wakefield and structure-based wakefield) [7,8], has generated strong interest in the generation of two closely spaced electron bunches ("twin bunches"), where two bunches are generated, accelerated and compressed in the same accelerating bucket $[9,10]$.

Twin bunches are applicable to $\mathrm{x}$-ray FELs for generating two-color $\mathrm{x}$-ray pulses with high power for time-resolved $\mathrm{x}$-ray pump/x-ray probe experiments. They can also be used in the fresh bunch scheme for seeded FELs to reduce spectral noise [11]. Moreover, the twin-bunch scheme is applicable to witness-bunch acceleration in plasma wakefield acceleration, as well as structure-based wakefield acceleration, which provides great flexibility in controlling the charge distribution and time delay, thus helping to stabilize the witness bunch at the best accelerating phase [7,12-14]. Finally, the twin-bunch method has also been used for two-color Thomson scattering sources [15].

\footnotetext{
*wangdanzei@gmail.com

yanlx@mail.tsinghua.edu.cn
}

Published by the American Physical Society under the terms of the Creative Commons Attribution 4.0 International license. Further distribution of this work must maintain attribution to the author(s) and the published article's title, journal citation, and DOI.
Taking X-FEL performance as an example, twin bunches with a tunable spacing (in both time and energy space) are desired to produce $\mathrm{x}$-rays with varying delays and wavelengths. In addition, a flexible time space up to picoseconds for twin electron bunches is important since electron dynamics typically occur on the scale of a few to tens of femtoseconds; reaching picosecond delays is crucial for the time-resolved investigation of nuclear motion in x-rayexcited samples [11]. Moreover, high-quality beams with unspoiled emittance from photoinjectors are also preferred for FEL lasing processes [16,17].

The longitudinal dynamics of twin bunches have been extensively discussed in the scientific literature, where the beam is compressed with 4-dipole chicane $[11,18]$ or with velocity bunching (VB) schemes [10,19]. In the VB scheme, the compression occurs along a rectilinear trajectory, free from coherent synchrotron radiation emission, which is the primary cause of emittance degradation in magnetic compressors [20-22]. Moreover, the VB scheme is generally associated with emittance compensation for low-energy beams, in which the beams emittance growth is optimized by tuning the long solenoids surrounding the accelerating section, thus maintaining the high-brightness beams from photoinjectors, which may be crucial for advanced applications in single-phase FELs and other advanced applications in wakefield accelerations $[23,24]$.

Twin-bunch compression by the VB scheme has been studied in Refs. [10,25,26]. Reference [26] has presented an optimized compression scheme for bunch train compression, called the deep overcompression mode of the VB 
scheme, where the phase of the bunch train injected into the TWA tube is set to $\ll-100$ degrees. The main benefits of this scheme are the relatively large phase acceptance and the uniformity of compression for the bunch train. Based on theory, in this paper, we investigate for the first time the generation of twin bunches with tunable spacing via the VB scheme by varying the TWA phase in a very large range. The results of both simulations and experiments are presented for a typical photoinjector, such as the Tsinghua Thomson $\mathrm{x}$-ray light source beamline (TTX) [27]. The results show that when the phase injected into the TWA tube is set to the deep overcompression mode phase, the generated twin bunches are continuously and widely tunable in time and energy space within a large TWA phase acceptance. In our case, the energy and time spaces can be adjusted continuously from 0 to $3 \mathrm{MeV}$ and from 0 to $6 \mathrm{ps}$, respectively, by varying the phase of the injected bunch train from -170 degrees to -120 degrees. In addition, simulations show that the emittance of each sub-bunch can also be preserved when the strength of the long solenoid surrounding the TWA is optimized for emittance compensation.

The remainder of this paper is organized as follows. We begin in Sec. II with basic considerations and detailed descriptions of the VB scheme, including the beamline parameters, different working modes of the VB scheme, and the emittance compensation scheme used in the simulations. In Sec. III, we present some typical simulation results for twin-bunch compression. In Sec. IV, experimental results for twin-bunch compression are presented and compared with the results of simulations. Finally, we provide concluding remarks in Sec. V.

\section{VELOCITY BUNCHING WITH THE TRAVELING WAVE ACCELERATOR}

The VB technique is a tool for compressing electron beams in modern high-brightness photoinjector sources [28-31]. The longitudinal phase space rotation in the VB process is based on a correlated time-velocity chirp in the electron bunch such that electrons on the tail of the bunch are faster than electrons in the bunch head. This rotation occurs inside the longitudinal potential of a traveling rf wave, which accelerates the beam inside a long multicell rf structure and simultaneously applies an off-crest energy chirp to the injected beam. This is possible if the injected beam is slightly slower than the phase velocity of the rf wave such that, when it is injected at the zero-crossing field phase, it slips back to phases where the field is accelerating but is simultaneously chirped and compressed. The key point is that compression and acceleration occur simultaneously within the same accelerator section, i.e., the initial section following the gun.

In an rf TWA structure, the electrons are subject to a longitudinal electric field, and the VB mechanism for an electron beam can be approximately expressed as [29]:

$$
\Delta \phi_{e}=\frac{\sin \phi_{0}}{\sin \phi_{e}} \Delta \phi_{0}+\frac{1}{2 \alpha \gamma_{0}^{2} \sin \phi_{e}} \Delta \gamma_{0}
$$

where $\phi_{0}$ and $\phi_{e}$ are the injection and exit phases of the reference particle with respect to the rf wave; $\Delta \phi_{0}, \gamma_{0}$ and $\Delta \gamma_{0}$ are the initial phase length, energy and energy spread, respectively; and $\Delta \phi_{e}$ is the phase length after compression. $\alpha=e E / m_{0} c^{2}$ is the normalized acceleration gradient, with $E$ being the peak field of the TWA, $k$ is the rf wave number, and $e, m_{0}$ and $c$ are constants representing the electron charge, the electron mass and the speed of light, respectively. Equation (1) evaluates the phase compression for a beam with a certain initial phase length and a certain energy spread.

We let $\sigma_{\phi_{0}}$ denote the initial rms phase (or rms bunch length) distribution of the beam at the entrance of the TWA and define the compression factor as follows:

$$
C=\sigma_{\phi_{e}} / \sigma_{\phi_{0}}
$$

Then, we can obtain the compression factor curves of $C$ versus $\phi_{0}$ for an electron beam with $\sigma_{\phi_{0}}=1$ degrees.

\section{A. Parameters of the beamline}

We study the VB scheme with the beamline at the Tsinghua University accelerator laboratory, which utilizes a typical setup for a photoinjector, as shown in Fig. 1. A 3 meter S-band SLAC-type traveling wave accelerator (TWA) is placed approximately 1.7 meters after a

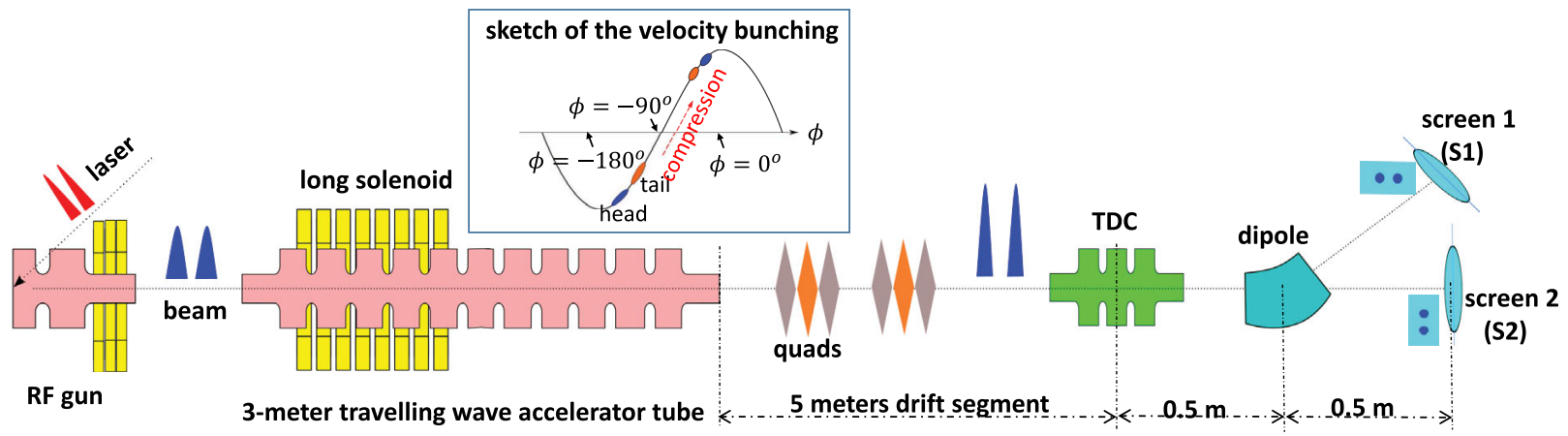

FIG. 1. Sketch of twin-bunch compression via VB at the TTX beamline. 
TABLE I. Parameters of the TTX beamline setup.

\begin{tabular}{ll}
\hline \hline Parameter & \multicolumn{1}{c}{ Value } \\
\hline Gradient of the rf gun & $120 \mathrm{MV} / \mathrm{m}$ \\
Phase of the rf gun & $30^{\circ}$ \\
Energy of the beam at the gun exit & $5.5 \mathrm{MeV}$ \\
Phase injected into the TWA $\left(\phi_{0}\right)$ & scanned parameter \\
Gradient of the TWA $(E)$ & $20 \mathrm{MV} / \mathrm{m}$ \\
Length of the downstream drift segment & $5 \mathrm{~m}$ \\
\hline \hline
\end{tabular}

BNL/KEK/SHI-type 1.6 cell S-band photocathode rf gun. The rf gun is set to a high gradient of $120 \mathrm{MV} / \mathrm{m}$, with a maximum acceleration phase of 30 degrees from the extraction phase, to boost the beam to relativistic energy in a short distance and reduce smearing effects due to space charges. For single electron bunch, the UV drive laser is Gaussian longitudinal distribution with rms length $1 \mathrm{ps}$, and radial uniform transverse distribution with radius $1.4 \mathrm{~mm}$. Thus, the initial state of the beam injected into the TWA is also defined. The TWA further accelerates the beam to $\sim 50 \mathrm{MeV}$ without compression. An energy spectrum (dipole magnet with YAG screen, S1) is used to diagnose the beam energy space, and a deflecting cavity with a YAG screen, S2, is used to diagnose the beam time space. Quadruple magnets in front are used to focus the beams into the deflecting cavity. The defecting cavity is 5 meters downstream of the TWA, and we set the defecting cavity position, i.e., beamline position $\approx 9.7$ meters, as a target position in our simulations. The operating parameters of the beamline are listed in Table I.

In a previous study [26], the TWA operated at a low gradient $(8 \mathrm{MV} / \mathrm{m})$ in deep over-compression mode. A relatively high gradient $(>10 \mathrm{MV} / \mathrm{m}$ ) can also be used to demonstrate the benefits of the deep over-compression mode in TWA, as shown in Fig. 2, which presents the compression factor versus TWA phase curves corresponding to different TWA gradients. The green dashed line denotes the case of no compression, where the compression factor $C=1 . E=10 \mathrm{MV} / \mathrm{m}$ (black curve) presents a

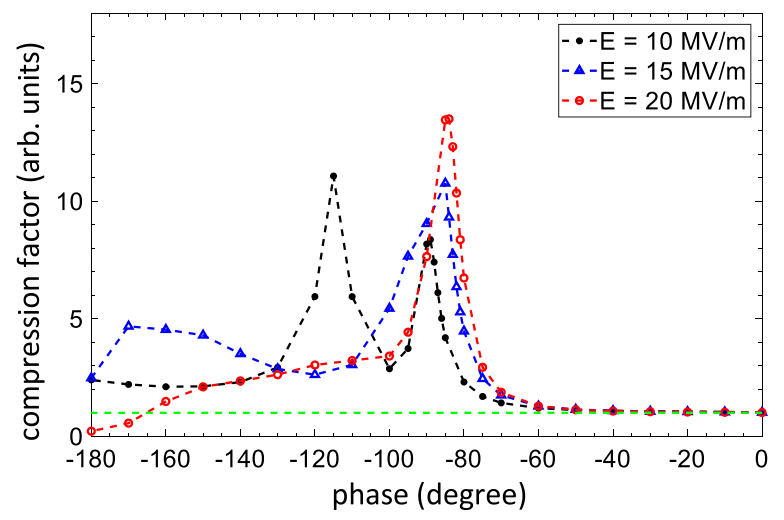

FIG. 2. Compression factor versus the TWA phases under different TWA gradients for single bunch. two-peak curve, as predicted in Ref. [26]. For the $E=$ $15 \mathrm{MV} / \mathrm{m}$ (blue curve) and $E=20 \mathrm{MV} / \mathrm{m}$ (red curve) cases, the second peak tends to be submerged into a flat and smooth bulge, and $C>1$ for phase $\ll-100$ degrees in a relatively large range. Thus, we choose the normal beamline operation gradient parameter of $20 \mathrm{MV} / \mathrm{m}$ (red curve in Fig. 2) in this study.

Note that in contrast to the low-gradient case in Ref. [26], the drift length downstream of the TWA is not an important parameter when choosing a relatively large gradient of the TWA because the energy space of the beam is fixed after the TWA at such a high gradient, even in the deep overcompression mode. Further accelerating the beam by other TWA tubes downstream, as in the INFN beamline [30], will not affect the presented results.

The curves in Fig. 2 are obtained from ASTRA simulations for a single electron beam with space charge effects [32]. The beam charge is $100 \mathrm{pC}$, and the initial bunch length is $1 \mathrm{ps}$. Notably, the compression factor curve with the space charge effect is relatively flat and smooth in the deep overcompression range compared with the sharp peak when the phase $=-90$ degrees. This observation indicates that the deep over-compression mode has two advantages: (a) there is no abrupt change in the compression factor as the injected phase is varied over a relatively large range, and (b) the compression is more uniform across all sub-bunches when the separation or distribution (i.e., the occupied range in the phase space) of the injected bunches is relatively large. Because of these beneficial characteristics, the deep overcompression mode is broadly applicable to twin-bunch compression with a wide variety of characteristics.

\section{B. Different compression modes in VB sheme}

VB can occur in several different compression modes depending on the phase injected into the TWA, as defined in Refs. [22,25]. As shown in Fig. 3, the different compression modes induced by different injected phases correspond to different variations in the rms bunch length along the beamline. The results are distinguished by differently colored curves (space charge effects are included here). In all cases, the initial injected rms bunch length before compression is 1 ps (black dashed line). In the full compression mode, corresponding to an injected phase of -90 degrees (red curve), the bunch length decreases to its minimum value at the TWA exit. With an injected phase of $>-90$ degrees ( -50 degrees, blue curve), the compression inside the TWA is insufficient, and some energy chirp remains in the beam at the TWA exit, causing the bunch length to continue decreasing in the drift segment downstream. In the over-compression mode, where the injected phase is $<-90$ degrees, and the deep overcompression mode when the injected phase is $\ll-90$ degrees $(-120$ degrees in the blue curve and -140 degrees in the magenta curve), the bunch length monotonically increases after the TWA, and the minimum bunch length 


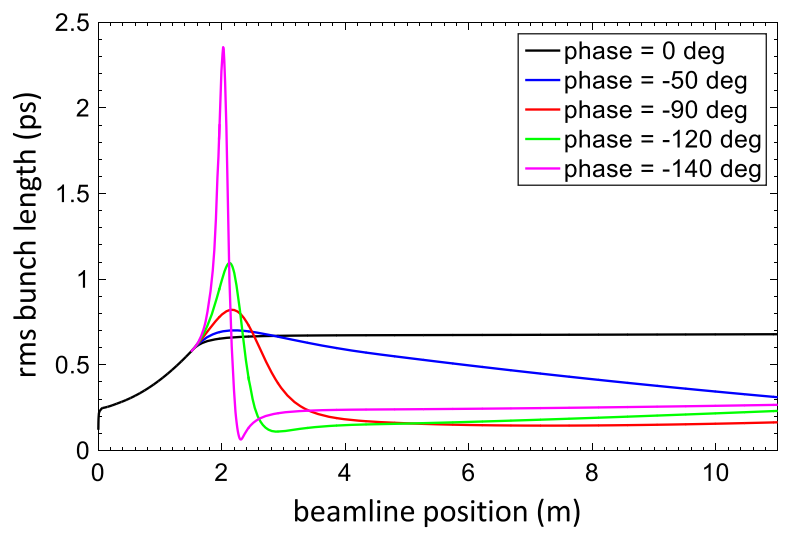

FIG. 3. Changes in rms bunch lengths along the beamline position for different TWA phases, and different phases correspond to different compression modes (no compression: $0 \mathrm{deg}$, under compression: $-50 \mathrm{deg}$, full compression: $-90 \mathrm{deg}$, and deep over-compression: $-120 \mathrm{deg}$ and $-140 \mathrm{deg}$ ).

value occurs inside the TWA. The bunch length after the TWA remains unchanged due to the relatively high gradient of the TWA, and the bunch lengths are relatively short.

\section{Emittance compensation in VB scheme}

To prevent irreversible emittance growth during bunch compression, the key issue is to preserve the laminarity of the beam with an envelope propagated as close as possible to a Brillouin-like flow. For the beams in VB, mismatches between the space-charge-correlated forces and the external focusing gradient produce slice envelope oscillations that cause normalized emittance oscillations. It has been shown that to keep such oscillations under control during VB, the beam has to be injected into the TWA with a laminar envelope waist $\left(\sigma^{\prime}=0\right)$, and the envelope has to be matched to the accelerating and focusing gradients such that it remains close to an equilibrium mode [23]. A long solenoid around the TWA is quite convenient to provide the necessary focusing.

In this configuration, the matching condition for the transverse rms envelope is given by

$$
\sigma_{e q}=\frac{1}{k} \sqrt{\frac{I_{0}}{4 \gamma_{0} I_{A}}\left(1+\sqrt{1+\left(\frac{4 \epsilon_{n} \gamma_{0} k I_{A}}{I_{0}}\right)^{2}}\right)}
$$

$I$ is the peak current in the beam; $k=e B_{0} / 2 m c$ is the transverse external focusing strength, where $B_{0}$ is the long solenoid field; $I_{A}=17 \mathrm{kA}$ is the Alfven current; and $\epsilon_{n}$ is the normalized emittance. The matching condition of Eq. (3) guarantees the preservation of the beam laminarity during acceleration and compression, but the final value of the emittance is strongly affected by the phase of the emittance oscillation in the VB, which cannot easily be predicted by theory. The emittance compensation could also be accomplished by fine tuning the beam size and long

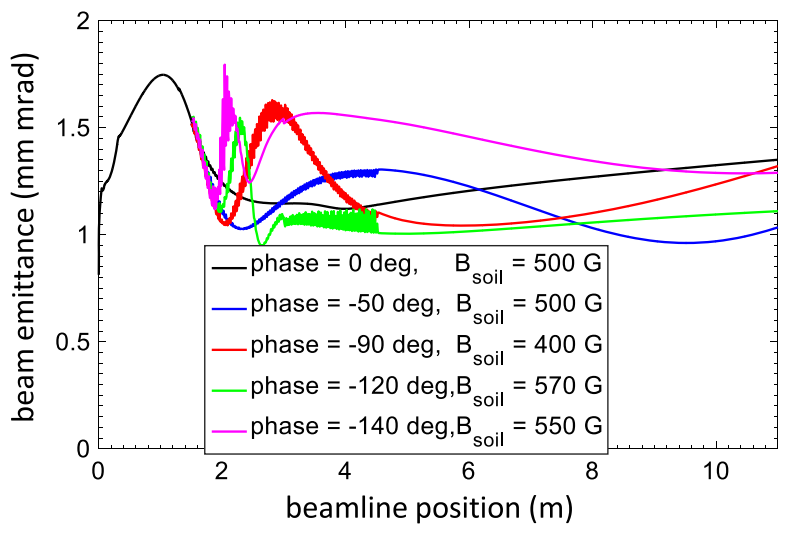

FIG. 4. Simulated emittance evolution along the beamline, with emittance compensation by tuning the long solenoid strength under different TWA phases.

solenoid fields, where the basic rule is to preserve transverse laminarity during bunch compression.

Figure 4 shows the simulated emittance evolution along the beamline, with emittance compensation achieved by tuning the long solenoid strength under different compression modes: no compression (phase $=0$ degrees, black curve) and undercompression mode (phase $=-70$ degrees, blue curve) with the long solenoids set to $500 \mathrm{G}$, the full-compression mode (phase $=-90$ degrees, red curve) with long solenoids set to $400 \mathrm{G}$, and deep overcompression mode (phase = -120 degrees and -140 degrees) with the solenoids set to $570 \mathrm{G}$ and $550 \mathrm{G}$. Note that the matching is not fully optimized in Fig. 4; the matching condition prescribed by Eq. (3) would indicate the optimized spot size at injection in the VB (at the beamline position $=170 \mathrm{~cm}$ ) after fine tuning the gun solenoid strength. However, the achieved envelopes in Fig. 4 already lead to relatively small emittances.

Note that the beam used in Fig. 4 are similar to the single bunch in the experiment below, and is close to the beam used in Ref. [23]. It's indeed not a very bright beam for no compression mode, with relatively low beam current around $30 \mathrm{~A}$, and relatively large beam emittance around $1.3 \mu \mathrm{m}$ (black line in Fig. 4). Because the initial laser spot size is relatively large, that lead to large initial emittance (a sharp rise at the beginning of curves in Fig. 4), besides, we didn't fully optimize the emittance by change other beamline parameters, tuning the long solenoid alone is not enough to optimize the beam emittance $[23,33]$. In the case of brighter beams, for example, when we change the laser spot size and the beamline parameters to get $100 \mathrm{pC}$ beam with $0.6 \mu \mathrm{m}$ emittance in no compression mode, then for the over-compression mode, we can get the similar trends of curves as magenta curve, red curve or green curve shown in Fig. 4, which means we can compensate linear emittance growth in the TWA, however, it is very hard to compensate the emittance below $0.8 \mu \mathrm{m}$. We observed silks in the phase space which caused by the strong nonlinear space charge effect and could not be compensate by solenoid. Because 
we inject beams into the TWA at the deceleration phase in overcompression mode, beams get decelerated and there is a longitudinal crossing of the beams, this high charge density will lead to emittance growth.

\section{SIMULATIONS OF TWIN-BUNCH COMPRESSION}

Twin bunches are generated with a two-bunch laser train illuminating a copper photocathode [34]. The initial bunch interval is $8 \mathrm{ps}$ in the simulations, and the charge of each sub-bunch is $100 \mathrm{pC}$.

Typical simulation results for twin-bunch compression with space charge effects are shown in Fig. 5. The projection onto the $\mathrm{x}$-axis is the beam energy, and the projection onto the y-axis is the time distributions. We use red and blue to represent bunches 1 and 2, respectively. The mean energy of the two bunches varies with the phase. When the phase $=0$ degrees, bunch 1 and bunch 2 are merged together in energy space, which is the case of minimal energy spread when twin bunches are on the rf crest in the TWA. In addition, from the time distributions, bunch 1 is in front of bunch 2 at injection, the rms bunch length of each sub-bunch and the bunch interval vary with the injected phase, and the two bunches are merged together in the time space when the phase $=-80$ degrees. Then, bunches 1 and 2 are reversed in the time space, i.e., bunch 1 is behind bunch 2 when the phase $<-80$ degrees. Note that the reference particle in the simulations for the twin bunches is located at the average position of the beam. Thus, the full-compression-mode phase of the twin bunches ( -80 degrees) corresponds to the single-bunch case when the phase $=-90$ degrees, as shown in Fig. 3.

Figure 6(a) shows more details of the twin-bunch compression when varying the TWA phase, including the average bunch length curve (red) and the average bunch emittance curve (blue). The bunch lengths of both bunches are compressed to relatively low values in the deep over-compression mode, where the TWA phase is in the range $[-170--120]$ degrees. In addition, the average bunch emittance is obtained with the emittance compensation scheme by adjusting the long solenoid strength when varying the TWA phase. The emittance values in different phases exhibit slight changes compared to the maximum acceleration case (phase $=0$ degrees), which means that the rf compression can effectively maintain the beam's emittance for advanced applications.

Figure 6(b) shows the bunch intervals and the energy spacing versus the injected phase. The initial interval and all intervals in the under-compression mode are positive, indicating that bunch 1 is in front of bunch 2, whereas negative values indicate the opposite case, with bunch 2 in front. The zero value in the bunch interval curves corresponds to the merged case (phase $=-80$ degrees). The accompanying values of the energy spacing between the two bunches have similar interpretations, i.e., positive values indicate that the average energy of bunch 1 is higher than that of bunch 2, whereas negative values indicate the opposite. We focus on the regions of phase -170 degrees to -120 degrees, where both the time and
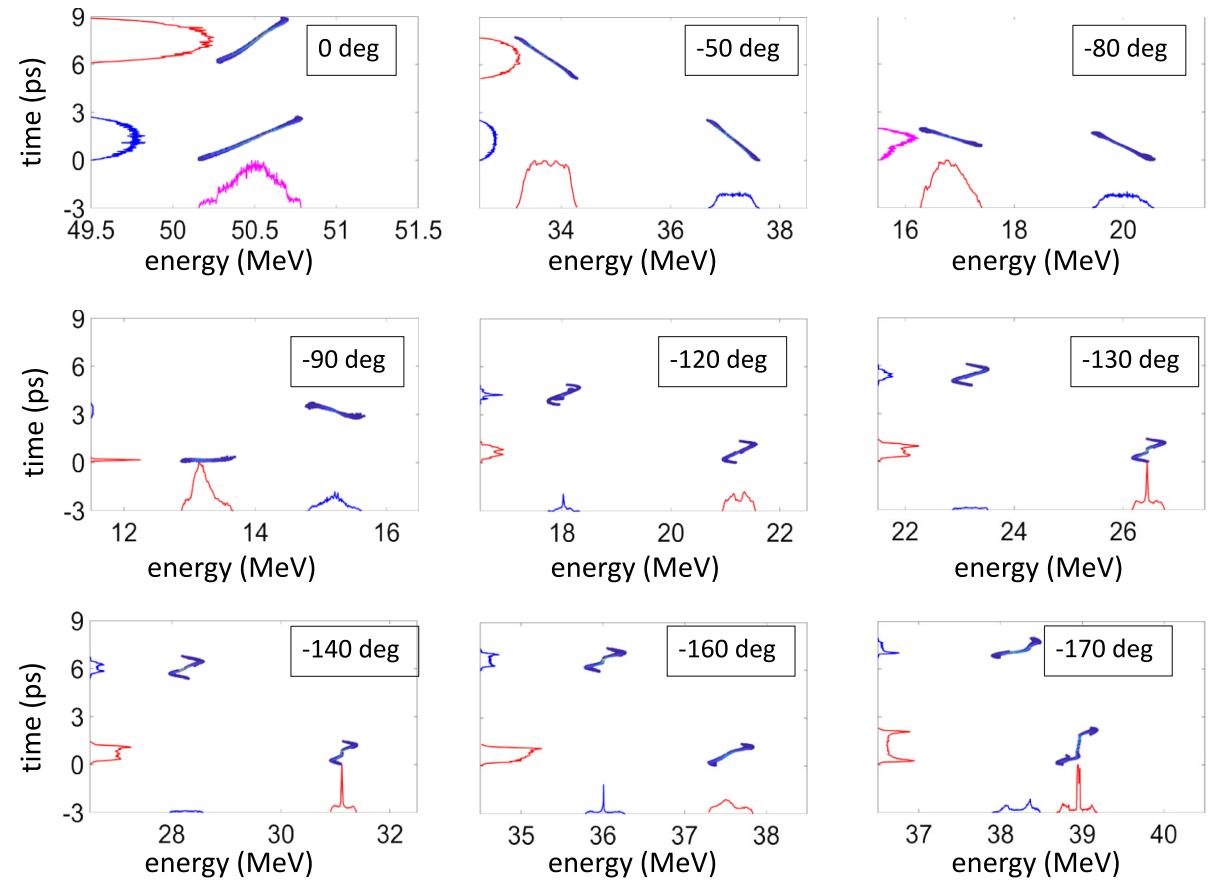

FIG. 5. Simulated phase space distributions of twin bunches (blue: bunch 1, red: bunch 2) under different TWA phases; the projection onto the $\mathrm{x}$-axis is the beam energy, and the projection onto the $\mathrm{y}$-axis is the time distributions. 


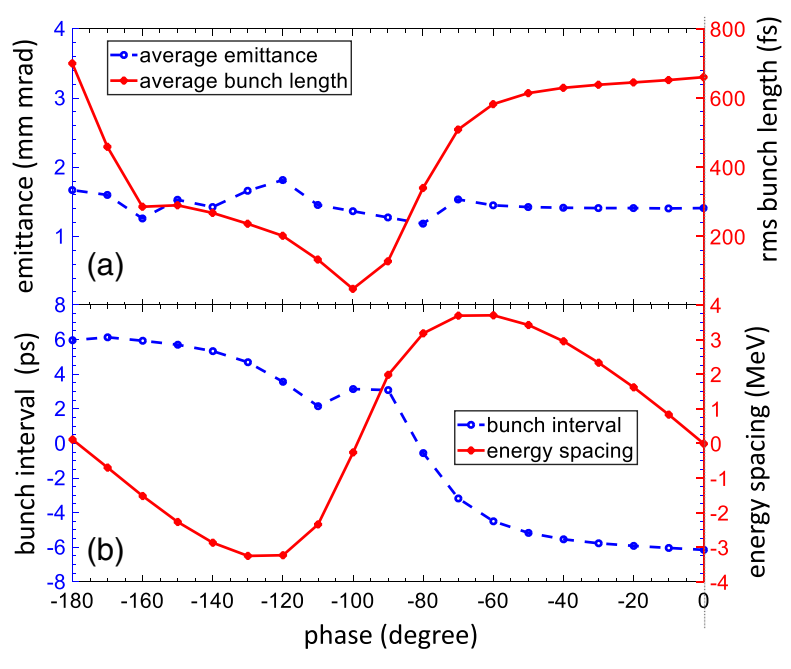

FIG. 6. ASTRA simulation results: (a) average bunch length and average bunch emittance versus the TWA phases, and (b) bunch intervals and the energy spacing versus the TWA phase.

energy spaces are continuously adjustable over quite a large range (up to $3 \mathrm{MeV}$ for the energy spacing between the two bunches and up to 6 ps for the time spacing). These results indicate the optimization of the twin bunches' tunability under the deep over-compression scheme.

Compared to other compression modes, the results also show the benefits of the deep over-compression scheme's (i) relatively short bunch length over the under-compression mode, such as in the phase $=-50$ degrees case; the (ii) relatively high energy compared to the full compression mode, as in the phase $=-80$ degrees case; and the (iii) relatively large phase acceptance of the TWA phase, from -170 degrees to -120 degrees.

\section{EXPERIMENTAL MEASUREMENTS}

The experimental setup is shown in Fig. 1, in which a dipole magnet with a YAG screen, S1, is used as the energy spectrum to measure the beam's energy, and a deflecting cavity with a YAG screen, S2, is used to measure the trains time space. Quadruple magnets downstream of the TWA are used to focus the beam into the deflecting cavity. The emittance measurement of bunches requires more quadrupoles and screens downstream the deflecting cavity [23], and we had plans to do it after the beamline updated.

In the presented beamline, bunches with different energies are separated horizontally by the dipole magnet, and bunches with different arrival times are separated vertically by the deflecting cavity. As shown in Fig. 7(a), the images on S1 with only the dipole magnet on show the beam's energy space, and in Fig. 7(b), the images on S2 with only the deflecting cavity on show the beam's time distribution. In addition, in Fig. 7(c), when we turn on both the dipole magnet and deflecting cavity only slightly, the images on S2 show both the energy and time spaces. Results in Fig. 7(a) and Fig. 7(b) are images from YAG screens, we only calibrate one axis of beam projection that we concern, i.e., energy of beam in Fig. 7(a) and time information in Fig. 7(b), the other axis without label shows the transverse beam size information on the YAG screen. The timescale and energy scale is not the same in Fig. 7(c) because we
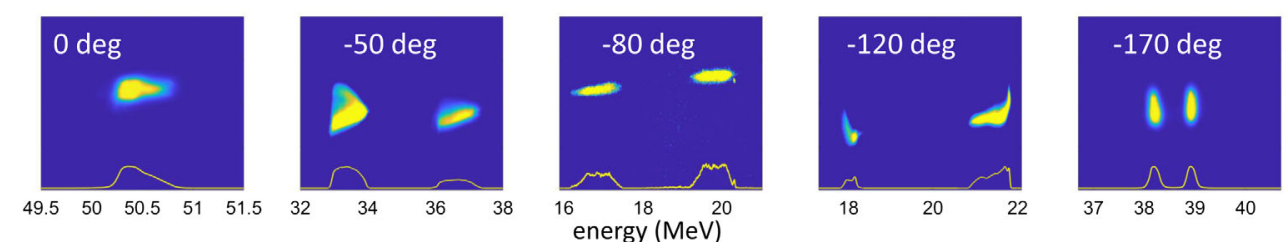

(a) images on S1 with dipole magnet on
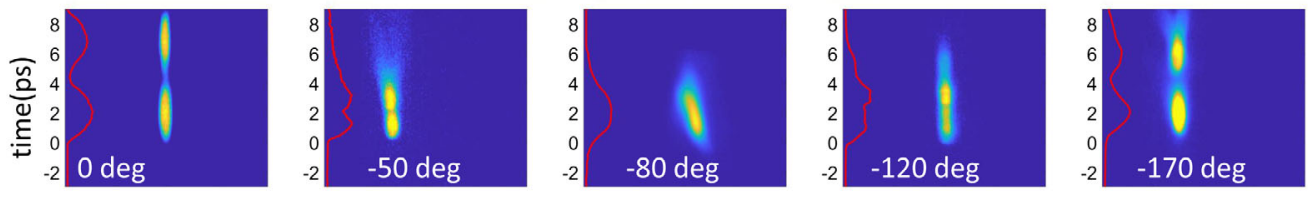

(b) images on S2 with deflecting cavity on
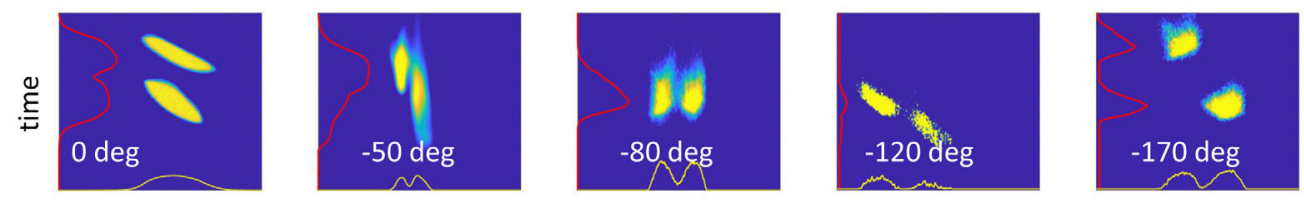

(c) images on S2 with dipole magnet and deflecting cavity on

FIG. 7. Experimental images used to measure the beam's energy and time spaces with different TWA phases: maximum acceleration when the phase $=0$ degrees, under-compression mode when the phase $=-50$ degrees, full-compression mode when the phase $=-80$ degrees, and deep over-compression modes when the phase $=-120$ degrees and the phase $=-170$ degrees . 


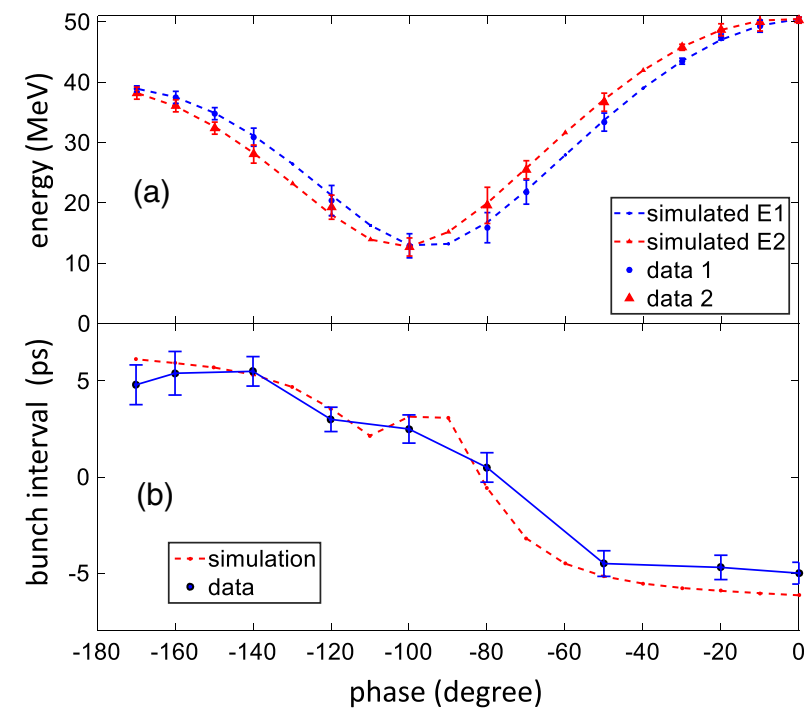

FIG. 8. Comparison between measured data and simulations: (a) energy of twin bunches under different TWA phases and (b) bunch intervals under different TWA phases.

have adjusted the strengths of both dipole magnet and deflecting cavity in order to get clear images on S2, thus they are only qualitative images to show the bunches' separation in energy and in time.

Because the beam's average energy varies with the TWA phase, we retune the beamline at different phases; we mainly adjust both the long solenoid strength to achieve emittance compensation and the quadrupoles to focus the beam into the deflecting cavity. The energy spectrum is calibrated with the dipole's current-magnetic field curve. The deflecting cavity is also calibrated with the voltageresolution curves at different beam energies.

We present five typical cases with different TWA phases in Fig. 7: the maximum acceleration case, where the phase $=0$ degrees; the under-compression case, where the phase $=-50$ degrees; the full-compression case, where the phase $=-80$ degrees; and the deep overcompression modes, where the phase $=-120$ degrees and the phase $=$ -170 degrees. The measurements are consistent with the ASTRA simulations in Fig. 5. We demonstrated that the deep over-compression mode of the VB scheme separated the beam in both time and energy, and it is greatly beneficial for twin-bunch compression due to its relatively large phase acceptance.

To provide a closer look at the compression properties, we show the measurement results compared with the simulation curve in Fig. 8. The energy of the twin bunches is shown in Fig. 8(a), and the bunch intervals are shown in Fig. 8(b). For data analysis, energy information is get from images as shown in Fig. 7(a). Time separation between two bunches is derived from images shown in Fig. 7(b), energy information is also used here because the energy is coupled into time separation measurement by deflecting cavity [35-37]. The measurements agree with the simulations, which demonstrate that the energy space and bunch intervals are tunable in the deep overcompression scheme in a relatively large range.

As mentioned in Ref. [26], the deep overcompression scheme is also suitable for multibunch train compression, where there are more than two sub-bunches in the train with picosecond intervals [38-42]. This utilizes the first TWA tube and the long solenoid around the TWA in typical photoinjectors, which makes it quite convenient to apply. In addition, it negates the need for a series of dipoles arranged in a chicane configuration, thus avoiding problems inherent to magnetic compression, such as momentum spread and transverse emittance dilution due to the bunch coherent synchrotron radiation. The main benefits of this scheme are the preservation of the beam's emittance, the relatively large phase acceptance and the uniformity of compression for the bunch train.

\section{SUMMARY}

In summary, we investigated the VB of twin bunches in a TWA through both simulations and experiments. We compressed the twin bunches by varying the TWA phase in a very large range. The results show that when the phase injected into the TWA is set to $\ll 100$ degrees, VB occurs in a deep overcompression mode, where the twin bunches are continuously and widely tunable within a large TWA phase acceptance. Moreover, the emittance of each subbunch is also preserved when the strength of the long solenoid surrounding the TWA is optimized, thus maintaining the high-quality beams from the photoinjector. The deep overcompression mode of the VB scheme for twin bunches is experimentally studied for the first time, herein achieving optimized operation for photoinjectors. This type of twin bunching generated via this optimized compression scheme, with charges of several hundred $\mathrm{pC}$, subpicosecond sub-bunch lengths and wide tunability in time and energy spaces, have great potential for application in scenarios involving FELs and advanced particle wakefield acceleration.

\section{ACKNOWLEDGMENTS}

This work was supported by the National Natural Science Foundation of China (NSFC Grant No. 11475097) and the National Key Scientific Instrument and Equipment Development Project of China (Grant No. 2013YQ12034504).

[1] A. Marinelli et al., High-intensity double-pulse X-ray freeelectron laser, Nat. Commun. 6, 6369 (2015).

[2] A. Lutman, R. Coffee, Y. Ding, Z. Huang, J. Krzywinski, T. Maxwell, M. Messerschmidt, and H.-D. Nuhn, Experimental Demonstration of Femtosecond Two-Color X-Ray Free-Electron Lasers, Phys. Rev. Lett. 110, 134801 (2013). 
[3] T. Hara, Y. Inubushi, T. Katayama, T. Sato, H. Tanaka, T. Tanaka, T. Togashi, K. Togawa, K. Tono, M. Yabashi et al., Two-colour hard X-ray free-electron laser with wide tunability, Nat. Commun. 4, 2919 (2013).

[4] V. Petrillo, M. Anania, M. Artioli, A. Bacci, M. Bellaveglia, E. Chiadroni, A. Cianchi, F. Ciocci, G. Dattoli, D. Di Giovenale et al., Observation of Time-Domain Modulation of Free-Electron-Laser Pulses by Multipeaked ElectronEnergy Spectrum, Phys. Rev. Lett. 111, 114802 (2013).

[5] G. De Ninno, B. Mahieu, E. Allaria, L. Giannessi, and S. Spampinati, Chirped Seeded Free-Electron Lasers: Self-Standing Light Sources for Two-Color Pump-Probe Experiments, Phys. Rev. Lett. 110, 064801 (2013).

[6] C. Ronsivalle, M. Anania, A. Bacci, M. Bellaveglia, E. Chiadroni, A. Cianchi, F. Ciocci, G. Dattoli, D. Di Giovenale, G. Di Pirro et al., Large-bandwidth two-color free-electron laser driven by a comb-like electron beam, New J. Phys. 16, 033018 (2014).

[7] M. Litos, E. Adli, W. An, C. Clarke, C. Clayton, S. Corde, J. Delahaye, R. England, A. Fisher, J. Frederico et al., High-efficiency acceleration of an electron beam in a plasma wakefield accelerator, Nature (London) 515, 92 (2014).

[8] B. O'Shea, G. Andonian, S. Barber, K. Fitzmorris, S. Hakimi, J. Harrison, P. Hoang, M. Hogan, B. Naranjo, O. Williams et al., Observation of acceleration and deceleration in gigaelectron-volt-per-metre gradient dielectric wakefield accelerators, Nat. Commun. 7, 12763 (2016).

[9] Z. Zhang, Y. Ding, A. Marinelli, and Z. Huang, Longitudinal dynamics of twin electron bunches in the Linac Coherent Light Source, Phys. Rev. Accel. Beams 18, 030702 (2015).

[10] R. Pompili, M. Anania, M. Bellaveglia, A. Biagioni, F. Bisesto, E. Chiadroni, A. Cianchi, M. Croia, A. Curcio, D. Di Giovenale et al., Beam manipulation with velocity bunching for PWFA applications, Nucl. Instrum. Methods Phys. Res., Sect. A 829, 17 (2016).

[11] Z. Zhang, Y. Ding, P. Emma, Z. Huang, A. Marinelli, and C. Tang, Flexible pulse delay control up to picosecond for high-intensity twin electron bunches, Phys. Rev. Accel. Beams 18, 090701 (2015).

[12] M. Hogan, T. Raubenheimer, A. Seryi, P. Muggli, T. Katsouleas, C. Huang, W. Lu, W. An, K. Marsh, W. Mori et al., Plasma wakefield acceleration experiments at FACET, New J. Phys. 12, 055030 (2010).

[13] D. Wang, X. Su, L. Yan, Y. Du, Q. Tian, Y. Liang, L. Niu, W. Huang, W. Gai, C. Tang et al., Phase control with two-beam interferometry method in a terahertz dielectric wakefield accelerator, Appl. Phys. Lett. 111, 174102 (2017).

[14] C. Jing, A. Kanareykin, J. Power, M. Conde, Z. Yusof, P. Schoessow, and W. Gai, Observation of Enhanced Transformer Ratio in Collinear Wakefield Acceleration, Phys. Rev. Lett. 98, 144801 (2007).

[15] V. Petrillo, A. Bacci, C. Curatolo, M. Ferrario, G. Gatti, C. Maroli, J. Rau, C. Ronsivalle, L. Serafini, C. Vaccarezza et al., Dual color $\mathrm{x}$ rays from Thomson or Compton sources, Phys. Rev. Accel. Beams 17, 020706 (2014).

[16] P. Emma, R. Akre, J. Arthur, R. Bionta, C. Bostedt, J. Bozek, A. Brachmann, P. Bucksbaum, R. Coffee, F.-J. Decker et al.,
First lasing and operation of an ångstrom-wavelength freeelectron laser, Nat. Photonics 4, 641 (2010).

[17] T. Ishikawa, H. Aoyagi, T. Asaka, Y. Asano, N. Azumi, T. Bizen, H. Ego, K. Fukami, T. Fukui, Y. Furukawa et al., A compact X-ray free-electron laser emitting in the subångström region, Nat. Photonics 6, 540 (2012).

[18] A. Marinelli, A. Lutman, J. Wu, Y. Ding, J. Krzywinski, H.-D. Nuhn, Y. Feng, R. Coffee, and C. Pellegrini, Multicolor Operation and Spectral Control in a GainModulated X-Ray Free-Electron Laser, Phys. Rev. Lett. 111, 134801 (2013).

[19] E. Chiadroni, M. Bellaveglia, P. Calvani, M. Castellano, L. Catani, A. Cianchi, G. Di Pirro, M. Ferrario, G. Gatti, O. Limaj et al., Characterization of the $\mathrm{THz}$ radiation source at the Frascati linear accelerator, Rev. Sci. Instrum. 84, 022703 (2013).

[20] L. Serafini and M. Ferrario, Velocity bunching in photoinjectors, AIP Conf. Ser. 581, 87 (2001).

[21] S. Anderson, P. Musumeci, J. Rosenzweig, W. Brown, R. England, M. Ferrario, J. Jacob, M. Thompson, G. Travish, A. Tremaine et al., Velocity bunching of high-brightness electron beams, Phys. Rev. Accel. Beams 8, 014401 (2005).

[22] D. Wang, L. Yan, Y. Du, J. Hua, Q. Du, H. Qian, X. Lu, W. Huang, H. Chen, and C. Tang, Generating ultrabroadband terahertz radiation based on the under-compression mode of velocity bunching, Rev. Sci. Instrum. 84, 022704 (2013).

[23] M. Ferrario, D. Alesini, A. Bacci, M. Bellaveglia, R. Boni, M. Boscolo, M. Castellano, E. Chiadroni, A. Cianchi, L. Cultrera et al., Experimental Demonstration of Emittance Compensation with Velocity Bunching, Phys. Rev. Lett. 104, 054801 (2010).

[24] R. Huang, Z. He, B. Li, S. Zhang, W. Li, Q. Jia, and L. Wang, Generation of high brightness electron beam by brake-applied velocity bunching with a relatively low energy chirp, Nucl. Instrum. Methods Phys. Res., Sect. A 866, 65 (2017).

[25] M. Ferrario, D. Alesini, A. Bacci, M. Bellaveglia, R. Boni, M. Boscolo, P. Calvani, M. Castellano, E. Chiadroni, A. Cianchi et al., Laser comb with velocity bunching: Preliminary results at SPARC, Nucl. Instrum. Methods Phys. Res., Sect. A 637, S43 (2011).

[26] D. Wang, L. Yan, Y. Du, W. Huang, W. Gai, and C. Tang, Theoretical analysis and simulation study of the deep overcompression mode of velocity bunching for a comblike electron bunch train, Phys. Rev. Accel. Beams 21, 024403 (2018).

[27] C. Tang, W. Huang, R. Li, Y. Du, L. Yan, J. Shi, Q. Du, P. $\mathrm{Yu}, \mathrm{H}$. Chen, T. Du, C. Cheng, and Y. Lin, Tsinghua Thomson scattering X-ray source, Nucl. Instrum. Methods Phys. Res., Sect. A 608, S70 (2009).

[28] T. Shintake and M. Ferrario, High performance electron injectors, Reviews of Accelerator Science and Technology (World Scientific, Singapore, 2010), Chap. 12, p. 221.

[29] P. Piot, L. Carr, W. S. Graves, and H. Loos, Subpicosecond compression by velocity bunching in a photoinjector, Phys. Rev. Accel. Beams 6, 033503 (2003).

[30] M. Boscolo, M. Ferrario, I. Boscolo, F. Castelli, and S. Cialdi, Generation of short $\mathrm{THz}$ bunch trains in a $\mathrm{RF}$ 
photoinjector, Nucl. Instrum. Methods Phys. Res., Sect. A 577, 409 (2007).

[31] B. Marchetti, M. Boscolo, M. Castellano, E. Chiadroni, M. Ferrario, B. Spataro, C. Vaccarezza, A. Cianchi, and I. T. Vergata, in Proceedings of the 2nd International Particle Accelerator Conference, San Sebastián, Spain (EPS-AG, Spain, 2011), THPS102, p. 3672.

[32] K. Floettmann, http://www.desy.de.

[33] Y. Ding, A. Brachmann, F.-J. Decker, D. Dowell, P. Emma, J. Frisch, S. Gilevich, G. Hays, P. Hering, Z. Huang et al., Measurements and Simulations of Ultralow Emittance and Ultrashort Electron Beams in the Linac Coherent Light Source, Phys. Rev. Lett. 102, 254801 (2009).

[34] L. Yan, Y. Du, C. Liao, J. Hua, Q. Du, W. Huang, and C. Tang, in Proceedings of the International Particle Accelerator Conference, Kyoto, Japan (ICR, Kyoto, 2010), WEPD051, p. 3210.

[35] L. Sabato, P. Arpaia, A. Giribono, A. Liccardo, A. Mostacci, L. Palumbo, C. Vaccarezza, and A. Variola, Effects of energy chirp on bunch length measurement in linear accelerator beams, Meas. Sci. Technol. 28, 084002 (2017).

[36] M. Huning, A. Bolzmann, H. Schlarb, J. Frisch, D. McCormick, M. Ross, T. Smith, J. Rossbach, U. Hamburg et al., Observation of femtosecond bunch length using a transverse deflecting structure, Stanford Linear Accelerator Center (SLAC) Technical Report No. SLAC-PUB-11482, 2005.
[37] Y. Ding, C. Behrens, P. Emma, J. Frisch, Z. Huang, H. Loos, P. Krejcik, and M. Wang, Femtosecond X-ray pulse temporal characterization in free-electron lasers using a transverse deflector, Phys. Rev. Accel. Beams 14, 120701 (2011).

[38] Y. Sun, P. Piot, A. Johnson, A. Lumpkin, T. Maxwell, J. Ruan, and R. Thurman-Keup, Tunable Subpicosecond Electron-Bunch-Train Generation Using a TransverseTo-Longitudinal Phase-Space Exchange Technique, Phys. Rev. Lett. 105, 234801 (2010).

[39] B. Jiang, J. G. Power, R. Lindberg, W. Liu, and W. Gai, Emittance-Exchange-Based High Harmonic Generation Scheme for a Short-Wavelength Free Electron Laser, Phys. Rev. Lett. 106, 114801 (2011).

[40] S. Antipov, M. Babzien, C. Jing, M. Fedurin, W. Gai, A. Kanareykin, K. Kusche, V. Yakimenko, and A. Zholents, Subpicosecond Bunch Train Production for a Tunable mJ Level THz Source, Phys. Rev. Lett. 111, 134802 (2013).

[41] P. Muggli, V. Yakimenko, M. Babzien, E. Kallos, and K. P. Kusche, Generation of Trains of Electron Microbunches with Adjustable Subpicosecond Spacing, Phys. Rev. Lett. 101, 054801 (2008).

[42] P. Musumeci, R. Li, and A. Marinelli, Nonlinear Longitudinal Space Charge Oscillations in Relativistic Electron Beams, Phys. Rev. Lett. 106, 184801 (2011). 